\title{
LA ACTUALIDAD DE LOS RITUALES AGRÍCOLAS MESOAMERICANOS. LA FIESTA DE LA SANTA CRUZ Y DE SAN ISIDRO LABRADOR EN DOS MUNICIPIOS MAZAHUAS DE MÉXICO
}

\author{
THE TRUTH ABOUT THE AGRICULTURAL RITUALS OF CENTRAL AMERICA. \\ THE SANTA CRUZ AND SAN ISIDRO LABRADOR CELEBRATIONS IN TWO \\ MAZAHUA TOWNSHIPS IN MEXICO
}

\author{
Vianney Maya*
}

\begin{abstract}
Mediante recursos documentales y arqueoastronómicos se sabe que las festividades asociadas con el cambio de los ciclos estacionales agrícolas son reconocidas como una de las más importantes de Mesoamérica, pues marcan el término de la sequía y el inicio de la temporada de lluvias desde la época prehispánica.

En este artículo, por medio del análisis de etnografía actual acerca de las fiestas de la Santa Cruz y de San Isidro Labrador en comunidades mazahuas de municipios de Ixtlahuaca y San Felipe del Progreso en México, se demuestra la pervivencia de rituales agrícolas como evidencia de un sincretismo identitario.
\end{abstract}

Palabras claves: Rituales agrícolas, fiesta de la Santa Cruz, fiesta de San Isidro Labrador, comunidades mazahuas.

According to documentaries and arqueological-astonomical resources, it is known that the festivities associated with the changes in seasonal agricultural cycles are recognized as the most important in Mesoamerica due to the fact that these mark the end of the dry season and the start of the rainy season from the beginning of the prehispanic period.

In this article, by way of the current analysis of ethnography in the Santa Cruz and San Isidro Labrador celebrations in Mazahua communities in Ixtlahuaca and San Felipe del Progreso in Mexico, the survival of the agricultural rituals are demonstrated as evidence and as an identifier of syncretism.

Key words: Agricultural rituals, Santa Cruz celebration, San Isidro Labrador celebration, Mazahua Communities.

\section{Introducción}

Para entender la visión y la construcción del mundo mesoamericano prehispánico debemos romper con ciertos esquemas que nos trajo la occidentalización, y anteponer la perspectiva de que las civilizaciones mesoamericanas concebían todas las esferas de la vida como un todo. Es decir, no estaban distanciadas las concepciones religiosas de las prácticas productivas y sociales. Sus estructuras económicas tenían como base la agricultura y esta se encontraba estrechamente asociada con su cosmovisión, regida al mismo tiempo por los ciclos naturales, contemplados de manera exacta en su medición calendárica.

Numerosos estudios dan cuenta de las asociaciones que tuvieron y han tenido hasta nuestros días los ciclos naturales con las creencias y prácticas religiosas en sociedades mesoamericanas. Partiendo de la "observación de la naturaleza" es que los nativos elaboraron complejas correspondencias. $\mathrm{La}$ medición del tiempo, el espacio estelar y terrestre, fenómenos atmosféricos, la orientación de centros urbanos y edificios ceremoniales, así como las prácticas económicas, mágicas y religiosas, eran correspondientes entre sí. Muestras de esta combinación nos brindan los trabajos en la especialidad de arqueoastronomía, que han sabido integrar metodológicamente disciplinas como la astronomía, la arqueología, la geografía, la antropología y la etnohistoria, comparando datos históricos con casos etnográficos actuales.

Partiendo de que el culto prehispánico mantiene una estrecha relación con los ciclos estacionales y agrícolas, el objetivo es ejemplificar con dos casos de comunidades campesinas mexicanas cómo sigue siendo latente, en combinación con el ejercicio de la religión católica, una concepción prehispánica en torno a la práctica ritual para propiciar el buen temporal.

\footnotetext{
* Escuela Nacional de Antropología e Historia, Ciudad de México, México. Correo electrónico: yennaivmaya@gmail.com
} 
Se hará referencia a dos comunidades en los municipios de Ixtlahuaca y San Felipe del Progreso, estado de México, pertenecientes a la etnorregión mazahua: San Pedro de los Baños y San Jerónimo Boncheté. En el primero se observa la fiesta que tiene lugar el 1 de mayo con motivo de la celebración del Día de la Cruz, mientras que en la segunda comunidad analizaremos cómo se lleva a cabo la celebración de San Isidro Labrador el 15 de mayo. La primera comunidad se distingue por tener un antecedente lacustre y ambas por basar su economía principalmente en la agricultura.

La información aquí vertida proviene de observaciones realizadas durante el ciclo ritual festivo en mayo de 2013 y 2014, así como por entrevistas abiertas y semiestructuradas realizadas a los habitantes de las comunidades en mención.

\section{El ciclo agrícola y los cultos campesinos en Mesoamérica}

Un planteamiento inicial para comprender la visión de los pueblos mesoamericanos subyace en el entendido de que el hombre ha establecido familiaridad con la naturaleza a partir de la interacción y la observación de la misma. De ahí su conocimiento cercano y la sacralidad con la que se dirige a esta. Por tanto, el paisaje, el territorio y la naturaleza han conformado, desde la época prehispánica, un sistema de creencias, significados y prácticas religiosas; es decir, parte fundamental de su cosmovisión (Broda 1991). Algunos de los estudiosos de la relación sociedad-naturaleza han sido Carlos García Mora, Johanna Broda y Raúl Aranda (De la Cruz Clemente 2014), quienes también precisan que esta relación no es inmutable, sino que va adquiriendo diversos cambios respecto de los procesos históricos que acontecen a cada comunidad.

Es útil precisar que en la actualidad comunidades campesinas mesoamericanas siguen distinguiendo espacios naturales sagrados dignos de respeto y de culto, de rituales de petición y de gratitud. Tales espacios son el cerro por excelencia, como proveedor de los mantenimientos; pero también lo son la cueva, los manantiales, los ojos de agua, las formaciones rocosas y la milpa. Estos referentes geográfico-simbólicos son parte fundamental de la cultura e identidad indígena, fundamento y destino de múltiples prácticas rituales y cotidianas (Broda y Gámez 2009).

Uno de los hallazgos más importantes de la arqueoastronomía es conocer la correlación existente entre el tiempo y el espacio de la cosmovisión mesoamericana. Muestra de ello son las orientaciones y las fechas en relación con la subdivisión del ciclo agrícola anual. Las prácticas ceremoniales más significativas se fechaban en momentos de cambio meteorológico, que marcaban la época de inicio de lluvias y de sequía. El culto a la lluvia se encuentra estrechamente relacionado con la agricultura, pues este recurso constituye una condición necesaria para el crecimiento de las plantas y el éxito de la producción agrícola (Broda1996:452).

Johanna Broda ha encontrado asociaciones entre fiestas de origen prehispánico y las que la etnografía actual nos brinda:

\section{"La fiesta mexicana del Huey tozoztli tenía lugar durante el apogeo de la estación seca y marcaba el tiempo para la siembra del maíz. Estos ritos prehispánicos encuentran su continuación hasta nuestros días en la fiesta de la Santa Cruz..." y agrega, "Su simbolismo sigue estando vinculado... con la petición de lluvia, la siembra del maíz y la fertilidad agrícola en general" (Broda 1996: 458).}

Pese al paso del tiempo, la imposición religiosa del catolicismo y el proceso sincrético, existe una tradición mesoamericana que sigue teniendo en claro la relación del culto a los espacios naturales y los fenómenos atmosféricos para el logro de sus cosechas. Las festividades con motivos agrícolas, por la implicación social que conllevan, manifiestan que "la religión es ante todo un sistema de acción, es vida social" (Broda 2001:166). Sin olvidar que estas manifestaciones de la religiosidad se encuentran en un "proceso creativo de reelaboración constante" (Broda 2001:167).

En nuestros días, son incontables las comunidades indígenas de México en las que el culto al agua y a la fertilidad agrícola sigue teniendo presencia, pues de la buena cosecha depende su sustento, es su modo de vida. Dos de las fiestas anuales más importantes de tradición indígena, a lo largo y ancho del territorio mexicano, son las que, al fusionarse con la evangelización cristiana, fueron destinadas para La Santa Cruz y San Isidro Labrador. Mismas que, por la fecha en las que se celebran, son marcadores temporales de la época en que termina la sequía para dar paso a las primeras lluvias. Junto con el Día de Muertos, estas celebraciones son 
consideradas como las fiestas más importantes de tradición indígena (Broda 2001:170).

\section{Ixtlahuaca y San Felipe del Progreso, dos municipios mazahuas del Estado de México}

Los casos de estudio son localizables en dos municipios del Estado de México, pertenecientes a la etnorregión mazahua: Ixtlahuaca y San Felipe del Progreso. Esta región limita al norte con el estado de Querétaro y con los municipios de Acambay y Timilpan del Estado de México; al sur con los municipios de Zinacantepec, Toluca, Amanalco de Becerra, Valle de Bravo e Ixtapan del Oro; al oriente con los municipios de Temoaya, Jiquipilco y Morelos; $\mathrm{y}$ al poniente con el municipio de Morelos en el estado de Michoacán (Comisión para el Desarrollo de los Pueblos Indígenas [CDI] 2013).

La lengua mazahua se deriva del tronco otopame, al que pertenece la familia otomí-mazahua. Se piensa que el grupo mazahua fue una de las cinco tribus chichimecas que migraron en el siglo XIII, encabezado por Mazahuatl, a quien se le debe este nombre y proveniente de los Acolhuas, llega a fundar el valle de Mazahuacan. Durante la consolidación del poderío azteca, Axayácatl sometió al grupo mazahua hacia el año de 1476 (Carrasco 1996). Más tarde, con la llegada de Gonzalo de Sandoval en la Colonia se introdujeron nuevas formas político-administrativas para el control de la tierra y la mano de obra, mediante la encomienda y la congregación, con lo que se tributaba a cambio de ser evangelizados por la orden franciscana.

En la actualidad la base de su economía sigue siendo la agricultura, principalmente del maíz; sin embargo la migración hacia la Ciudad de México y Toluca, así como hacia Estados Unidos, ha ganado terreno como parte de su sistema económico.

Ixtlahuaca de Rayón como oficialmente se reconoce, en 2010 registraba 141.482 habitantes, de estos 54.450 son de origen indígena, con un grado de marginación medio. En tanto que San Felipe del Progreso tiene 121.396, de ellos 76.627 son indígenas, y según los indicadores sociodemográficos de la población total y la población indígena 2010, posee un alto grado de marginación (Comisión para el Desarrollo de los Pueblos Indígenas [CDI] 2013).

Los dos municipios que comprenden las comunidades de estudio se ubican geográficamente en la parte norte del Estado de México y son colindantes entre sí. Ixtlahuaca, limita al poniente con San Felipe del Progreso.

\section{La Fiesta de la Santa Cruz en San Pedro de los Baños, Ixtlahuaca}

En San Pedro de los Baños, Ixtlahuaca, el primer día de mayo tiene lugar la celebración del Día de la Santa Cruz, a diferencia de otras comunidades mexicanas que la celebran el tres de mayo. Esta peculiaridad es referida al hecho de que en esta fecha se colocó la primera piedra de la capilla donde celebran a su afamada y milagrosa Cruz de piedra. La gente entrevistada asegura que esta Cruz apareció dentro de la laguna, ahora desecada, que albergaba aguas termales, muy concurridas por sus propiedades curativas.

A esta celebración acuden pueblos vecinos devotos, principalmente La Concepción de los Baños, comunidad con la que históricamente San Pedro de los Baños se ha disputado la propiedad de la laguna. De manera extraordinaria, a raíz de la pérdida del agua, que fue entubada en la década de 1970 para proveer a la Ciudad de México, estas comunidades se han unido de manera ritual para reconocerse mediante la Cruz como una sociedad lacustre.

Sobresalen a lo largo del día las danzas de "los comanches" o "concheros", "las pastoras", "la danza de Santiagos" y un contingente de hombres con calzón y camisa de manta, que ejecutan tambores de cuero y silbatos denominados los tita jucha. Tanto la presencia de pastoras como de los tita jucha, danzas regionales tradicionales, tienen asociación con gestos propiciatorios de lluvia. El bastón de las pastoras es símbolo de fertilidad; además, los cascabeles que usan las primeras, así como los silbatos y tambores que ejecutan los segundos, aluden al sonido de la lluvia y el trueno.

La procesión que llega a la capilla en el momento cumbre de la celebración es encabezada por mujeres que ofrecen manojos de flores rojas y ceras, se trata de las mayordomas de San Pedro, quienes aseguran que las flores son producto de la tierra y al mismo tiempo eso es lo que se pretende, que la tierra siga beneficiándolos con sus frutos.

Durante la celebración católica el sacerdote pide por las lluvias, buenas cosechas, salud y bienestar de la gente de San Pedro y de sus animales. Al término de esta los mayordomos reparten a los asistentes gladiolas rojas y ceras, estas últimas pueden ser encendidas en situaciones difíciles o 
ante una enfermedad a lo largo del año, es una especie de "protección" o "ayuda" que brinda la Cruz y los Santitos.

Frente a la capilla, en un campo que fuera parte de la laguna, se destina un espacio para realizar las "carreras de caballos", mejor conocidas como "cortaderas". Se trata de una competencia entre dos jinetes a la vez en una distancia de aproximadamente trescientos metros. Los corredores participantes llegan a alcanzar el centenar y a todos ellos se le gratifica obsequiándoles un "pollo", gallina, gallo o guajolote que la misma comunidad ha donado. Estos deben correr con su ave en alto, sujetándola con una mano. Anteriormente los dos jinetes competidores se disputan un ave al mismo tiempo que corrían, esta práctica es ya poco vista en la actualidad. Pues el objetivo final de esta disputa era que el ave muriera degollada.

La sangre del ave que se vertía sobre la tierra bien pudo cumplir la función de un ritual de sacrificio donde se pretende alimentar a la tierra, para que, de manera recíproca, la tierra brindara el alimento a los hombres por medio de sus productos agrícolas y los animales que se nutren de ella.

Los participantes de esta práctica no asocian la muerte del ave con un ritual de sacrificio para la tierra, la laguna o los santos, pero reconocen que la sangre aviva los ánimos de los asistentes, "le da más emoción". Además de que consideran al ave obsequiada como "una bendición para sus corralitos". Si esta no es sacrificada durante las cortaderas, se le cuida con especial atención y no se come, pues se cree que esta hará que sus demás aves se reproduzcan. Como cierre de esta celebración, los mayordomos ofrecen comida a todos los asistentes a manera de convite colectivo.

\section{Fiesta de San Isidro Labrador en San Jerónimo Boncheté}

La fiesta de San Isidro Labrador en San Jerónimo Boncheté, municipio de San Felipe del Progreso, da inicio el 14 de mayo por la noche con la llegada de la banda de viento y la elaboración de los báculos, conformados por una estructura de madera. Llama la atención el hecho de que sean mujeres quienes porten este símbolo de empoderamiento y asociación directa con la capacidad creadora, pues son justamente ellas quienes tienen la capacidad, al igual que la tierra, de dar vida. El ornamento del cual están cubiertas las estructuras de madera son guías de palomitas de maíz; cuando se les cuestiona sobre el porqué de las palomitas de maíz responden que son alimento, se comen, vienen de la tierra, son del maíz.

Al día siguiente, 15 de mayo, la banda y los cohetes dan la bienvenida al día, para que más tarde se incorporen los grupos de danza, generalmente "pastoras" y "arrieras". Al término de la misa católica del medio día, en las afueras de la iglesia, el sacerdote realiza una bendición rociando agua, a todos los tractores, caballos y yuntas que participan de las labores del campo y que han sido ornamentados con guías, banderas de colores y globos. Con este fin se han reunido todos los campesinos. Primeramente se bendice a las yuntas, en seguida los tractores, las camionetas, e incluso se han introducido entre las filas un contingente de niños en bicicletas, carros particulares y taxis, como parte de la concepción de que con estos vehículos de servicio también se gana el sustento. Algunas mayordomas van sahumando el camino, otras portan canastas de flores y ceras, a las que denominan "ofrendas" y así llevan en procesión a San Isidro Labrador por todo el poblado.

$\mathrm{Al}$ término de la caminata, se llega a un terreno donde los asistentes se instalan, las yuntas por un lado, los tractores por otro, y al centro del terreno se coloca a San Isidro. Teniendo por testigo al santo, se inician "las limpias" a las yuntas, los tractores y demás vehículos. Esta consiste en pasar por encima de estos a manera de barrido una cera y un manojo de hierbas, con la intención de que este gesto simbólico limpie de toda desgracia sus instrumentos agrícolas y reciban solo bendiciones en el nuevo ciclo que comienza; es decir, se realice una renovación anual y se garantice de esta manera el éxito en las actividades campesinas. Como pago por la limpia que promueven los mayordomos, cada participante da en "ofrenda" una pequeña cantidad de dinero destinado a mejoras de la iglesia.

Otra de las prácticas que se realizan a manera de ofrenda o acto ritual son las regionalmente famosas carreras de caballos o cortaderas. Estas a diferencia de las de San Pedro de los Baños no son tan numerosas y cuentan con menos aves para gratificar a los corredores.

Las danzas se han hecho presentes, los grupos femeninos de pastoras y arrieras se han rotado para presentarse frente a San Isidro, hasta el momento en el que se lleva a cabo el cambio de mayordomos. Los mayordomos salientes hacen entrega de una cruz pequeña de madera a los mayordomos 
entrantes como "símbolo del compromiso" que están adquiriendo para las fiestas de enero a mayo del próximo año. Este hecho marca el término de un ciclo anual festivo, a la vez que precisa el inicio del ciclo anual agrícola.

Al finalizar este acto todos emprenden nuevamente el regreso a la iglesia para depositar a San Isidro y continuar a las afueras con la comida que algunas mayordomas han llevado para todos los asistentes, nuevamente el realizar un banquete denota la culminación del acto ritual del que todos participan.

\section{Conclusiones}

A lo largo de esta exposición se dio muestra de la actualidad de las prácticas rituales que tienen lugar durante las fiestas religiosas en dos comunidades del centro de México, pertenecientes a la etnorregión mazahua. Estas prácticas son resultado de una tradición que ha sabido integrar elementos culturales mesoamericanos y cristianos, como parte de la cosmovisión que posee este grupo étnico. Contemplando cosmovisión como "la visión estructurada en la cual las nociones cosmológicas eran integradas en un sistema coherente" (Broda 1996).

La relación que aún hasta nuestros días poseen las festividades del Día de la Santa Cruz y el día de San Isidro Labrador y las fechas prehispánicas en las que se celebraban los ritos agrícolas, es un referente para subrayar la correspondencia que los pueblos campesinos siguen teniendo con la naturaleza y su entorno mediante actos de reciprocidad.
La laguna y la milpa como espacios sagrados es donde confluye la gente año con año para seguir realizando sus peticiones por un buen temporal. El sacrificio de aves, el uso del sahumerio y el copal, la ofrenda de flores y ceras, la limpia de los instrumentos de trabajo y los báculos como símbolos de fertilidad, son indicadores de la pervivencia de un pensamiento precristiano que se ha fundido con el culto a los santos y las representaciones católicas. Estos rituales de naturaleza agraria son un canal de comunicación para agradar a los espacios naturales y a sus santos que fungen como custodios de los recursos, como proveedores. De no darse esta continuidad ritual, la vida agrícola peligraría y, por tal razón, la de toda la comunidad campesina.

Las prácticas rituales aquí vertidas son parte de la memoria colectiva de estas dos comunidades mazahuas, como parte del pensamiento continuo; es decir, viven en la consciencia del grupo que las lleva a cabo, y aunque existe una permanencia esencial, se adaptan según los momentos y procesos históricos, los contextos locales y globales.

Pese al avance de la homogeneización promovida por la era global, existen elementos de marcado arraigo en todas las sociedades, ya que en estos se sustenta su identidad y cercanía con su entorno. Los pueblos mesoamericanos encuentran sustento material e ideológico en su práctica agrícola, es la lluvia y la benevolencia climática que proveerá de maíz al pueblo, por consiguiente, quienes cultivan la tierra se deben a los rituales por medio de los cuales se pretende propiciar el buen temporal.

\section{Referencias Citadas}

Broda, Johanna

1991 Cosmovisión y observación de la naturaleza: el ejemplo del culto a los cerros en Mesoamérica. En Arqueoastronomía, y etnoastronomía en Mesoamérica, editado por Johanna Broda, Stanislaw Iwaniszewski y Lucrecia Maupomé (eds.), pp. 461500. Universidad Nacional Autónoma de México, México.

Broda, Johanna

1996 Calendarios, cosmovisión y observación de la naturaleza. En Temas mesoamericanos, editado por Sonia Lombardo y Enrique Nalda (coords.), pp. 427-469. Instituto Nacional de Antropología e Historia, Dirección General de Publicaciones del Consejo Nacional para la Cultura y las Artes, México.

Broda, Johanna

2001 La etnografía de la fiesta de la Santa Cruz: una perspectiva histórica. En Cosmovisión, ritual e identidad de los pueblos indígenas de México, editado por Consejo Nacional para la Cultura y las Artes - Fondo de Cultura Económica, pp. 165-232. México D.F.
Broda, Johanna

2004. Ciclos agrícolas en la cosmovisión prehispánica: el ritual mexica. En Historia y vida ceremonial en las comunidades mesoamericanas: los ritos agrícolas, editado por Johanna Broda y Catharine Good (coords.), pp. 35-81. Instituto Nacional de Antropología e Historia, Universidad Nacional Autónoma de México, México, D.F.

Broda, Johanna

2009 Religiosidad popular y cosmovisiones indígenas en la Historia de México. INAH, México D.F.

Broda, Johanna y Félix Báez Jorge (coords.)

2001 Cosmovisión, ritual e identidad de los pueblos indígenas de México. Consejo Nacional para la Cultura y las Artes - Fondo de Cultura Económica, México D.F.

Broda, Johanna y Alejandra Gámez (coords.)

2009 Cosmovisión mesoamericana y ritualidad agrícola. Estudios interdisciplinarios y regionales. Benemérita Universidad Autónoma de Puebla, Puebla, México. 
De la Cruz Clemente, $\mathrm{P}$.

2014 Menye, el espíritu del agua y la laguna en la historia propia de un pueblo mazahua, San Pedro de los Baños, Ixtlahuaca, México (1936-2014). Tesis para optar por el título de Maestría en Antropología Social, Escuela Nacional de Antropología e Historia, México.

Good, Catharine

2001 El ritual y la reproducción de la cultura: ceremonias agrícolas, los muertos y la expresión estética entre los nahuas de Guerrero. En Cosmovisión, ritual e identidad de los pueblos indígenas de México. Consejo Nacional para la Cultura y las Artes - Fondo de Cultura Económica, pp. 239-297. México D.F.
CDI.

2014 Indicadores sociodemográficos de la población total y población indígena 2010, Cédulas de información básica de los pueblos indígenas de México 2010 de Ixtlahuaca. http://www.cdi.gob.mx/cedulas/2010/MEXI/15042-10.pdf. (15 octubre 2014).

CDI

2014 Indicadores sociodemográficos de la población total y población indígena 2010, Cédulas de información básica de los pueblos indígenas de México 2010 de San Felipe del Progreso. http://www.cdi.gob.mx/cedulas/2010/MEXI/1507410.pdfv. (15 octubre 2014). 